\title{
Quantum retrodiction in open systems
}

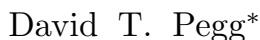 \\ School of Science, Griffith University, Nathan, Brisbane 4111, Australia \\ Stephen M. Barnetti and John Jeffers \\ Department of Physics and Applied Physics, University of Strathclyde, Glasgow G4 ONG, Scotland
}

(Dated: February 1, 2008)

\begin{abstract}
Quantum retrodiction involves finding the probabilities for various preparation events given a measurement event. This theory has been studied for some time but mainly as an interesting concept associated with time asymmetry in quantum mechanics. Recent interest in quantum communications and cryptography, however, has provided retrodiction with a potential practical application. For this purpose quantum retrodiction in open systems should be more relevant than in closed systems isolated from the environment. In this paper we study retrodiction in open systems and develop a general master equation for the backward time evolution of the measured state, which can be used for calculating preparation probabilities. We solve the master equation, by way of example, for the driven two-level atom coupled to the electromagnetic field.
\end{abstract}

PACS numbers: 03.67.Hk, 03.65.Wj,42.50.-p

\section{INTRODUCTION}

The usual formulation of quantum mechanics is predictive. It is well suited for calculating probabilities for particular outcomes of measurements from given preparation information. The preparation information is normally incorporated into a density operator representing the state of the prepared system, which evolves until the time of measurement. With sufficient knowledge we can assign a density operator in the form of a pure state projector to the system. This contains the maximum amount of information that nature allows us for prediction. The probability of the measurement outcome also depends on the operation of the measurement device. For a von Neumann measurement [1] a particular measurement outcome is associated with a pure state projector; for a more general measurement the outcome is associated with an element of a probability operator measure (POM) 22]. The latter are non-negative definite operators that sum to the unit operator on the state space of the system being measured. The retrodictive formalism of quantum mechanics is used far less often than the predictive formalism. Originally introduced by Aharonov et al. [3] in investigating the origin of the arrow of time, the retrodictive formalism involves assigning a state to the system based on knowledge of the measurement outcome. This state is assigned to the system just prior to the measurement and evolves backwards in time to the preparation event. The probability of a particular preparation outcome can be calculated from a knowledge of the backwards evolved state and the operation of the preparation device. While the results obtained are fully con-

\footnotetext{
D.Pegg@sct.gu.edu.au

tsteve@phvs.strath.ac.uk

损ohn@phys.strath.ac.uk
}

sistent with predictive quantum mechanics plus inference based on Bayes' theorem [1], the retrodictive approach is much more direct and provides a different insight into quantum mechanics.

Most applications of retrodiction involve closed systems, where the time evolution is essentially unitary [4. 5]. An important emerging area in which retrodictive quantum mechanics will become increasingly important is in quantum communication [6], including quantum cryptography [7]. Here a sender transmits a system to a recipient after preparing it in a particular state. The general communication problem is for the recipient to retrodict the prepared state from the outcome of a measurement and a knowledge of the operation of the preparation device. For closed systems, the evolution between preparation and measurement is unitary and the intrinsic time symmetry significantly simplifies the problem of calculating the retrodictive evolution. In practice, however, realistic systems will interact with an environment into which information is irretrievably lost. This introduces extra uncertainty in addition to the intrinsic quantum uncertainty associated with the preparation and measurement and removes the simple time symmetry associated with unitary evolution in a closed system. In open systems with a large environment the predictive evolution can often be described by a master equation. This describes information loss as the system propagates forward in time and becomes more entangled with an environment that is never measured. In this paper we investigate the derivation of a retrodictive master equation [8] that describes irreversible loss of information as the system propagates backwards in time. As will be seen, this is not a simple time inverse of the predictive master equation. 


\section{PREPARATION AND MEASUREMENT WITHOUT TIME DELAY}

The probabilistic interpretation of quantum mechanics is usually expressed in terms of the theory of measurement. Retrodiction, however, relies on preparation being probabilistic so, in this section, we give a brief overview of the less familiar quantum theory of preparation and measurement. To dispense with time evolution at this stage, we consider an experiment in which one person, the preparer, prepares a quantum system and then another person, the measurer, immediately measures it. We assume that there are readout mechanisms on the preparation and measurement devices that indicate the preparation event and the measurement event that occur. The preparer chooses which preparation event will occur and the measurer chooses whether or not to record the subsequent measurement event depending on what this measurement event is. If the measurement event $j$ is recorded, then so too is the corresponding preparation event $i$. These events are recorded as a combined event $(i, j)$. The experiment is repeated many times with the preparer choosing various states and a list of recorded combined events is made. The probability associated with a particular combined event on the list is defined in the usual way as the suitably normalized occurrence frequency. Both preparer and measurer have some control over the statistics, which contain elements of preselection and postselection.

The connection with quantum mechanics can be incorporated in the basic symmetric expression [9 for the probability that a combined event chosen at random on the list is $(i, j)$ :

$$
P^{\Lambda \Gamma}(i, j)=\frac{\operatorname{Tr}\left(\hat{\Lambda}_{i} \hat{\Gamma}_{j}\right)}{\operatorname{Tr}(\hat{\Lambda} \hat{\Gamma})}
$$

where

$$
\hat{\Gamma}=\sum_{j} \hat{\Gamma}_{j}
$$

and

$$
\hat{\Lambda}=\sum_{i} \hat{\Lambda}_{i}
$$

Here $\hat{\Lambda}_{i}$ and $\hat{\Gamma}_{j}$ are non-negative definite operators associated with the preparation and measurement events $i$ and $j$ respectively. They indicate the state in which the system is prepared or measured as well as any bias in preparation or in recording the events [9]. The sums in (2) and (3) are over respectively the measurement and preparation events that can be recorded. We say that the set of preparation device operators $\hat{\Lambda}_{i}$ describes mathematically the operation of the preparation device and the set of measurement device operators $\hat{\Gamma}_{j}$ describes the operation of the measurement device. Later, in equation (16), we shall see that $\hat{\Lambda}_{i}$ is $P^{\Lambda}(i) \hat{\rho}_{i}^{\text {pred }}$ where $\hat{\rho}_{i}^{\text {pred }}$ is the density operator for the prepared state and $P^{\Lambda}(i)$ is the a priori probability for this state to be prepared, which reflects any bias in the preparer's choice. Thus $\hat{\Lambda}$ is a density operator representing the best description we can give of the state in which the system is prepared if we know the operation of the preparation device but have no knowledge of the preparation or measurement events. Likewise $\hat{\Gamma}$ is a density operator representing the best description we can give of the state in which the system is measured if we know the operation of the measurement device but have no knowledge of the preparation or measurement events.

\section{A. Preparation and measurement probabilities}

The basic relation (1) allows us to find expressions for various probabilities [9] in terms of preparation and measurement device operators $\hat{\Lambda}_{i}$ and $\hat{\Gamma}_{j}$. We use superscripts $\Lambda$ and $\Gamma$ to denote if the probabilities are based on knowledge of the operations of the preparation and measurement devices respectively. Where a probability is based on knowledge of a particular preparation event $i$ or measurement event $j$, this is shown in the argument of the probability in the usual way by a vertical stroke preceding $i$ or $j$ respectively. This stroke essentially means 'if'. A comma separating events means 'and'. Thus, for example, $P^{\Lambda \Gamma}(j \mid i)$ is the probability that measurement event $j$ is recorded if preparation event $i$ is recorded, based on knowledge of the operations of the preparation and measurement devices.

From the basic relation (1), with (2) and (3), we can calculate the following probabilities:

$$
P^{\Lambda \Gamma}(i)=\sum_{j} P^{\Lambda \Gamma}(i, j)=\frac{\operatorname{Tr}\left(\hat{\Lambda}_{i} \hat{\Gamma}\right)}{\operatorname{Tr}(\hat{\Lambda} \hat{\Gamma})}
$$

$$
P^{\Lambda \Gamma}(j)=\frac{\operatorname{Tr}\left(\hat{\Lambda} \hat{\Gamma}_{j}\right)}{\operatorname{Tr}(\hat{\Lambda} \hat{\Gamma})}
$$

$$
P^{\Lambda \Gamma}(j \mid i)=\frac{P^{\Lambda \Gamma}(i, j)}{P^{\Lambda \Gamma}(i)}=\frac{\operatorname{Tr}\left(\hat{\Lambda}_{i} \hat{\Gamma}_{j}\right)}{\operatorname{Tr}\left(\hat{\Lambda}_{i} \hat{\Gamma}\right)}
$$

$$
P^{\Lambda \Gamma}(i \mid j)=\frac{\operatorname{Tr}\left(\hat{\Lambda}_{i} \hat{\Gamma}_{j}\right)}{\operatorname{Tr}\left(\hat{\Lambda} \hat{\Gamma}_{j}\right)}
$$

Expression (4) is the probability that, if an experiment chosen at random has a recorded combined event, this event includes preparation event $i$. Likewise (5) is the probability that the recorded combined event includes the measurement event $j$. Expression (6) is the probability that, if the recorded combined event includes event $i$, then it also includes event $j$. Expression (6) can be obtained by limiting the sample space to those events 
containing $i$ and is essentially Bayes' formula 10. Likewise (7) is the probability that the preparation event in a recorded combined event was $i$ if the measurement event is $j$.

It is clear that we can multiply all the $\hat{\Lambda}_{i}$ by a constant value without changing the above probabilities and similarly for $\hat{\Gamma}_{j}$. We make use of this flexibility to set

$$
\operatorname{Tr} \hat{\Lambda}=1
$$

and

$$
\operatorname{Tr} \hat{\Gamma}=1
$$

thus giving these operators the same normalization as density operators.

Expression (6) can be used for prediction. We can calculate the required probability if we know the preparation device operator (PDO) $\hat{\Lambda}_{i}$ associated with the preparation event $i$ and if we know the mathematical description of the operation of the measuring device, that is, every measurement device operator (MDO) $\hat{\Gamma}_{j}$. Similarly we can use (7) for retrodiction from our knowledge of $\hat{\Gamma}_{j}$ and all the $\hat{\Lambda}_{i}$ of the preparation device.

In the situation where no measurement is made on the system, there is only one possible measurement outcome and we can speak in terms of an effective measurement device whose operation is represented by a single 'noinformation' MDO that is proportional to the unit operator 99. Substituting this MDO for $\hat{\Gamma}_{j}$ in (7) yields the retrodictive probability $P^{\Lambda \Gamma}(i \mid j)$, which we write in this case as $P^{\Lambda}(i)$, where

$$
P^{\Lambda}(i)=\operatorname{Tr} \hat{\Lambda}_{i}
$$

Expression (10), which depends only on the operation of the preparation device, is what we would normally call the a priori probability for the preparation event to be $i$, that is, the probability in the absence of knowledge of the preparation outcome, the measurement outcome and the operation of the measurement device.

\section{B. Biased devices}

Consider the special case where the operation of a measurement device is such that the probability $P^{\Lambda \Gamma}(i)$ that a recorded preparation event is $i$ in the absence of knowledge of the preparation or measurement outcomes is equal to the a priori probability $P^{\Lambda}(i)$ for $i$. Then, from (4) and (10),

$$
\frac{\operatorname{Tr}\left(\hat{\Lambda}_{i} \hat{\Gamma}\right)}{\operatorname{Tr}(\hat{\Lambda} \hat{\Gamma})}=\operatorname{Tr} \hat{\Lambda}_{i}
$$

If this is true for all possible preparation devices, that is for all $\hat{\Lambda}_{i}$ and $\hat{\Lambda}$, then from (8) $\hat{\Gamma}$ must be proportional to the unit operator acting on the space of the system. We say that such measurement devices, which faithfully preserve the a priori probabilities of preparation events in the record, have an unbiased operation. Thus for an unbiased measuring device operation we can write

$$
\hat{\Gamma}=G \hat{1}
$$

where $G$ is a positive constant and $\hat{1}$ is the unit operator. We then define

$$
\hat{\Pi}_{j}=\hat{\Gamma}_{j} / G
$$

which allows us to write (6) as

$$
P^{\Lambda \Gamma}(j \mid i)=\operatorname{Tr}\left(\hat{\rho}_{i}^{\mathrm{pred}} \hat{\Pi}_{j}\right)
$$

where

$$
\hat{\rho}_{i}^{\text {pred }}=\hat{\Lambda}_{i} / \operatorname{Tr} \hat{\Lambda}_{i}
$$

This is just the usual, that is predictive, density operator that we assign to the prepared state on the basis of knowledge of the preparation event. We note from (12) and (13) that $\hat{\Pi}_{j}$ are also non-negative definite and sum to the unit operator. Thus $\hat{\Pi}_{j}$ form the elements of a probability operator measure (POM) [2]. Expression (14), which applies to unbiased measurement devices, is sometimes regarded as the fundamental postulate for the probabilistic interpretation of quantum mechanics [2]. As we shall see later, even when the operation of the measurement device is biased, it is still convenient sometimes to use $\hat{\rho}_{i}^{\text {pred }}$ as defined by (15) and in this case the predictive probability (6) becomes (27) instead of (14). Equations $(15)$ and (10) give an expression for $\hat{\Lambda}_{i}$ in terms of the predictive density operator associated with preparation event $i$ and the a priori probability that this state is prepared:

$$
\hat{\Lambda}_{i}=P^{\Lambda}(i) \hat{\rho}_{i}^{\text {pred }}
$$

This allows us to interpret $\hat{\Lambda}$ in (3) as just the predictive density operator that we would assign to the prepared state if we knew the operation of the preparation device but had no knowledge of the measurement or of the preparation outcome.

Likewise, in the situation where we know only the system state space and are totally ignorant of the preparation we are able to represent the operation of the preparation device by a single 'no-information' $\mathrm{PDO}$ that is proportional to the unit operator. Substituting this PDO for $\hat{\Lambda}_{i}$ in (6) yields

$$
P^{\Gamma}(j)=\operatorname{Tr} \hat{\Gamma}_{j}
$$

We shall call expression (17), which depends only on the operation of the measurement device, the a priori probability for the measurement event to be $j$, that is, the probability in the absence of knowledge of the measurement outcome and any preparation information.

$P^{\Lambda \Gamma}(j)$ is the probability that a recorded event is $j$ if the operations of the preparation and measurement 
devices are known but the actual preparation event is not known. From (5) and (17) this will be equal to the a priori probability for $j$ if

$$
\frac{\operatorname{Tr}\left(\hat{\Lambda} \hat{\Gamma}_{j}\right)}{\operatorname{Tr}(\hat{\Lambda} \hat{\Gamma})}=\operatorname{Tr} \hat{\Gamma}_{j}
$$

If this is true for all possible measurement devices, that is for all $\hat{\Gamma}_{j}$ and $\hat{\Gamma}$, then $\hat{\Lambda}$ must be proportional to the unit operator acting on the space of the system. We say that such preparation devices, which faithfully preserve the $a$ priori probabilities of measurement events in the record, have an unbiased operation. The retrodictive probability (7) reduces for an unbiased preparation device operation to

$$
P^{\Lambda \Gamma}(i \mid j)=\operatorname{Tr}\left(\hat{\rho}_{j}^{\mathrm{retr}} \hat{\Xi}_{i}\right) .
$$

where $\hat{\Xi}_{i}$ sum to the unit operator and are the preparation POM elements, which are proportional to $\hat{\Lambda}_{i}$, and

$$
\hat{\rho}_{j}^{\text {retr }}=\hat{\Gamma}_{j} / \operatorname{Tr} \hat{\Gamma}_{j} .
$$

The symmetry between retrodictive and predictive expressions is evident. It is worth remarking, however, that the usual experimental situation is asymmetric. This arises not from any intrinsic asymmetry in quantum mechanics but from the asymmetry in the operations of normal preparation and measurement devices. The operation of the latter can usually be described by a POM and so (14) is applicable; the former is usually not described by a POM so we must use the more general formula (7) and not (19). This also results in an asymmetry between the forms of $P^{\Lambda \Gamma}(i)$ and $P^{\Lambda \Gamma}(j)$ in that $P^{\Lambda \Gamma}(i)$ is just the a priori probability $P^{\Lambda}(i)$ in (10), which is independent of the operation of the measurement device, but $P^{\Lambda \Gamma}(j)$ does depend on the operation of the preparation device. It is convenient, therefore, to express $P^{\Lambda \Gamma}(j)$ in terms of $P^{\Lambda}(i)$. The expression for this is

$$
P^{\Lambda \Gamma}(j)=\sum_{i} P^{\Lambda \Gamma}(j \mid i) P^{\Lambda}(i) .
$$

Thus when the operation of the measuring device is unbiased, Bayes' theorem [11], for example, can be written in terms of the a priori probability $P^{\Lambda}(i)$ as

$$
P^{\Lambda \Gamma}(i \mid j)=\frac{P^{\Lambda \Gamma}(j \mid i) P^{\Lambda}(i)}{\sum_{i} P^{\Lambda \Gamma}(j \mid i) P^{\Lambda}(i)} .
$$

The concept of an unbiased operation of a preparation or measurement device is of particular relevance for practical cases. For an unbiased operation of a measurement device, the occurrence frequency of the preparation event $i$ in the list of combined events is proportional to the $a$ priori probability for $i$. An unbiased measuring device allows the preparer to control the relative occurrence frequency of a preparation event. Likewise, an unbiased operation of a preparation device allows the occurrence frequency of the measurement event $j$ to be proportional to the a priori probability for $j$, where this probability is based on knowledge of the operation of the measurement device but no knowledge of the actual measurement outcome or of the operation of the preparation device. An unbiased preparation device allows the measurer to control the occurrence frequency of the measurement event. In practice the operations of many, but not all, measuring devices are unbiased but preparation devices generally have biased operations. This corresponds to preselection without postselection. For such cases, as outlined above, we can write the predictive probability for measurement outcome $j$ given preparation event $i$ in terms of a predictive density operator and an element of a probability operator measure (POM) for the measuring device as given by (14). That is, for a measuring device with an unbiased operation, the preparation device operator $\hat{\Lambda}_{i}$ converts to a density operator and the measurement device operator $\hat{\Gamma}_{j}$ converts to a POM element. We cannot in general, however, write the corresponding retrodictive probability as the inverse expression involving an element of a probability operator measure for the preparation device and a retrodictive density operator. To do this we would need the operation of the preparation device to be unbiased, as occurs in some situations such as the Bennett-Brassard protocol for quantum key distribution [4, ㄱ, 12.

\section{TIME EVOLUTION IN CLOSED SYSTEMS}

So far we have considered the preparation time $t_{p}$ to be the same as the measurement time $t_{m}$ and in (11) we have used $\hat{\Lambda}_{i}$, the preparation device operator at $t_{p}$. To allow now for time evolution in a closed system between $t_{p}$ and $t_{m}$ we replace $\hat{\Lambda}_{i}$ and $\hat{\Lambda}$ in the probability (1) by $\hat{\Lambda}_{i}\left(t_{m}\right)$ and $\hat{\Lambda}\left(t_{m}\right)$ where

$$
\hat{\Lambda}_{i}\left(t_{m}\right)=\hat{U}\left(t_{m}, t_{p}\right) \hat{\Lambda}_{i} \hat{U}^{\dagger}\left(t_{m}, t_{p}\right)
$$

and a corresponding expression for $\hat{\Lambda}\left(t_{m}\right)$. Here $\hat{U}\left(t_{m}, t_{p}\right)$ is the unitary time-shift operator. We might interpret this replacement as a modification of the original operation of our preparation device to include an evolutionary period so that the new preparation device operators are $\hat{\Lambda}_{i}\left(t_{m}\right)$. The resulting probability expressions obtained from (1) will be modified accordingly. Thus the predictive expression (6) becomes

$$
P^{\Lambda \Gamma}(j \mid i)=\frac{\operatorname{Tr}\left[\hat{U}\left(t_{m}, t_{p}\right) \hat{\Lambda}_{i} \hat{U}^{\dagger}\left(t_{m}, t_{p}\right) \hat{\Gamma}_{j}\right]}{\operatorname{Tr}\left[\hat{U}\left(t_{m}, t_{p}\right) \hat{\Lambda}_{i} \hat{U}^{\dagger}\left(t_{m}, t_{p}\right) \hat{\Gamma}\right]}
$$

and the retrodictive expression (7) becomes

$$
P^{\Lambda \Gamma}(i \mid j)=\frac{\operatorname{Tr}\left[\hat{U}\left(t_{m}, t_{p}\right) \hat{\Lambda}_{i} \hat{U}^{\dagger}\left(t_{m}, t_{p}\right) \hat{\Gamma}_{j}\right]}{\operatorname{Tr}\left[\hat{U}\left(t_{m}, t_{p}\right) \hat{\Lambda} \hat{U}^{\dagger}\left(t_{m}, t_{p}\right) \hat{\Gamma}_{j}\right]} .
$$

From (15), noting that $\operatorname{Tr} \hat{\Lambda}_{i}$ is unchanged by a unitary transformation, we see (23) is equivalent to the usual 
time development expression

$$
\hat{\rho}_{i}^{\text {pred }}\left(t_{m}\right)=\hat{U}\left(t_{m}, t_{p}\right) \hat{\rho}_{i}^{\text {pred }}\left(t_{p}\right) U^{\dagger}\left(t_{m}, t_{p}\right)
$$

where $\hat{\rho}_{i}^{\text {pred }}\left(t_{p}\right)$ is the usual, that is predictive, density operator for the prepared state at $t_{p}$. Using (15) we can rewrite (24) as

$$
P^{\Lambda \Gamma}(j \mid i)=\frac{\operatorname{Tr}\left[\hat{\rho}_{i}^{\text {pred }}\left(t_{m}\right) \hat{\Gamma}_{j}\right]}{\operatorname{Tr}\left[\hat{\rho}_{i}^{\text {pred }}\left(t_{m}\right) \hat{\Gamma}\right]} .
$$

A natural interpretation of (27) is in terms of the system being prepared in the state $\hat{\rho}_{i}^{\mathrm{pred}}\left(t_{p}\right)$ at $t_{p}$ and then evolving forwards in time to become $\hat{\rho}_{i}^{\text {pred }}\left(t_{m}\right)$ at the measurement time.

At first sight, it may appear that the evolution has destroyed the symmetry between (16) and (7) which have now become (24) and 25). However by using the cyclic property of the trace we rewrite both (24) and (25) in terms of $\hat{\Gamma}_{j}\left(t_{p}\right)$ where

$$
\hat{\Gamma}_{j}(t)=\hat{U}^{\dagger}\left(t_{m}, t\right) \hat{\Gamma}_{j} \hat{U}\left(t_{m}, t\right),
$$

with $t_{p} \leq t \leq t_{m}$. Then, for example, the retrodictive formula (25) becomes

$$
P^{\Lambda \Gamma}(i \mid j)=\frac{\operatorname{Tr}\left[\hat{\Lambda}_{i} \hat{\Gamma}_{j}\left(t_{p}\right)\right]}{\operatorname{Tr}\left[\hat{\Lambda} \hat{\Gamma}_{j}\left(t_{p}\right)\right]} .
$$

Expressions (28) and (29) can be interpreted in terms of a new operation of the measurement device which incorporates a prior time delay as part of the measurement process. This operation is described by the set of measurement device operators $\hat{\Gamma}_{j}\left(t_{p}\right)$.

We can use (28) and (20) to write (29) as

$$
P^{\Lambda \Gamma}(i \mid j)=\frac{\operatorname{Tr}\left[\hat{\Lambda}_{i} \hat{\rho}_{j}^{\text {retr }}\left(t_{p}\right)\right]}{\operatorname{Tr}\left[\hat{\Lambda} \hat{\rho}_{j}^{\text {retr }}\left(t_{p}\right)\right]}
$$

where

$$
\hat{\rho}_{j}^{\mathrm{retr}}\left(t_{p}\right)=\hat{U}^{\dagger}\left(t_{m}, t_{p}\right) \hat{\rho}_{j}^{\mathrm{retr}}\left(t_{m}\right) \hat{U}\left(t_{m}, t_{p}\right) .
$$

Expressions (30) and (31) can be interpreted as the system being assigned a retrodictive state $\hat{\rho}_{j}^{\text {retr }}\left(t_{m}\right)$ at time $t_{m}$ on the basis of the recorded measurement outcome and evolving backwards in time to the preparation time $t_{p}$.

Any of the above four interpretations of the process of preparation and measurement can be applied to both the predictive and retrodictive probabilities (24) and (25). It is not necessary, for example, to use the predictive density operator for prediction, nor the retrodictive density operator for retrodiction. Use of the forward-evolved predictive density operator is convenient, however, when we know the outcome of a particular preparation and wish to retain flexibility in calculating probabilities for a range of possible measurement events with different measuring devices. Likewise, if we know the measurement outcome and wish to have flexibility in calculating probabilities for a range of possible preparation events with different preparation devices, it is convenient to calculate the backward-evolved retrodictive density operator. This is the purpose of this paper, except instead of finding the unitary evolution for a closed system, we wish to study the non-unitary evolution of a more realistic open system.

\section{OPEN SYSTEMS}

In a preliminary paper [8] we outlined the derivation of an open system retrodictive master equation for the particular case where the operation of the measurement device can be described by a POM. In this section we examine in detail the more general case where the set of (renormalized) measurement device operators may not necessarily form a POM and also give a proof of the nonnegativity of the retrodictive density operator at all times between measurement and preparation. Later we solve the master equation for a driven two-level atom.

We are interested in a quantum system $S$ interacting with an environment $E$. The initial state of the environment at time $t_{p}$ is known but the state of the environment subsequent to this is never measured. Before examining the retrodictive problem we outline first the usual predictive problem. In the predictive problem, in addition to the initial state of $E$, we also know the initial state of $S$ and wish to predict the probabilities for the outcomes $j$ of possible later measurements of $S$. For this we can use expressions (27) and (26) where $\hat{\rho}_{i}^{\text {pred }}\left(t_{p}\right)$ is now the predictive density operator for the combined system of $E$ and $S$, which will be the product of the initial density operators for $E$ and $S$ :

$$
\hat{\rho}_{i}^{\text {pred }}\left(t_{p}\right)=\hat{\rho}_{E}^{\text {pred }} \otimes \hat{\rho}_{i, S}^{\text {pred }} .
$$

This initial state will evolve to a state which is in general entangled in accord with (26) where the unitary operator will now involve the Hamiltonian of the combined system. Each measurement device operator will be a product an operator $\hat{\Gamma}_{j, S}$ acting on $S$ and an operator acting on the environment. As discussed earlier, the measurement device operator for a non-measurement is proportional to the unit operator as this is effectively an unbiased measurement with only one possible outcome 9 . Thus the predictive probability (27) becomes for this case

$$
P^{\Lambda \Gamma}(j \mid i)=\frac{\operatorname{Tr}_{E S}\left[\hat{\rho}_{i}^{\text {pred }}\left(t_{m}\right) \hat{\Gamma}_{j, S} \otimes \hat{1}_{E}\right]}{\operatorname{Tr}_{E S}\left[\hat{\rho}_{i}^{\text {pred }}\left(t_{m}\right) \hat{\Gamma}_{S} \otimes \hat{1}_{E}\right]} .
$$

where $\hat{1}_{E}$ is the unit operator on the space of the the environment and $\hat{\Gamma}_{S}$ is the sum of $\hat{\Gamma}_{j, S}$. The trace is over the environment and the system $S$ states. By defining a reduced predictive density operator for the system $S$ at the time $t$ as 13

$$
\hat{\rho}_{i, S}^{\text {pred }}(t)=\operatorname{Tr}_{E}\left[\hat{U}\left(t, t_{p}\right) \hat{\rho}_{E}^{\text {pred }} \otimes \hat{\rho}_{i, S}^{\text {pred }} \hat{U}^{\dagger}\left(t, t_{p}\right)\right]
$$


we can write the predictive probability in terms of operators acting on the state space of $S$. Substituting (26), (32) and (34) with $t=t_{m}$ into (33) gives

$$
P^{\Lambda \Gamma}(j \mid i)=\frac{\operatorname{Tr}_{S}\left[\hat{\rho}_{i, S}^{\text {pred }}\left(t_{m}\right) \hat{\Gamma}_{j, S}\right]}{\operatorname{Tr}_{S}\left[\hat{\rho}_{i, S}^{\text {pred }}\left(t_{m}\right) \hat{\Gamma}_{S}\right]},
$$

which is of the same form as the closed system formula (27). Thus in order to find the predictive probability $P^{\Lambda \Gamma}(j \mid i)$ we need only calculate the evolution of the reduced predictive density operator rather than the evolution of the combined system plus environment. If the environment $E$ has a large number of degrees of freedom and is little changed by the coupling to $S$, then if the environment is not measured we can make use of the approximation that, for any time $t$ between preparation and measurement 13,

$$
\hat{U}\left(t, t_{p}\right) \hat{\rho}_{E}^{\text {pred }} \otimes \hat{\rho}_{i, S}^{\text {pred }} \hat{U}^{\dagger}\left(t, t_{p}\right) \approx \hat{\rho}_{E}^{\text {pred }} \otimes \hat{\rho}_{i, S}^{\text {pred }}(t) .
$$

The Markov approximation 13 then leads to a general Markovian master equation for $\hat{\rho}_{i, S}^{\text {pred }}(t)$ that, in standard Lindblad form, is given by 14

$$
\begin{aligned}
\dot{\hat{\rho}}_{i, S}^{\text {pred }}(t)= & -i \hbar^{-1}\left[\hat{H}_{S}, \hat{\rho}_{i, S}^{\text {pred }}(t)\right] \\
& +\sum_{q}\left[2 \hat{A}_{q} \hat{\rho}_{i, S}^{\text {pred }}(t) \hat{A}_{q}^{\dagger}-\hat{A}_{q}^{\dagger} \hat{A}_{q} \hat{\rho}_{i, S}^{\text {pred }}(t)\right. \\
& \left.-\hat{\rho}_{i, S}^{\text {pred }}(t) \hat{A}_{q}^{\dagger} \hat{A}_{q}\right]
\end{aligned}
$$

where $\hat{H}_{S}$ is the Hamiltonian for the system $S$ without the environment and $\hat{A}_{q}$ is a system operator. This general form of equation incorporates the Markovian requirement and conserves both the non-negative definiteness and the trace of the reduced density operator [14].

The expression for the retrodictive probability (29) is

$$
P^{\Lambda \Gamma}(i \mid j)=\frac{\operatorname{Tr}_{E S}\left[\hat{\Lambda}_{i, S} \otimes \hat{\rho}_{E}^{\text {pred }} \hat{\Gamma}_{j}\left(t_{p}\right)\right]}{\operatorname{Tr}_{E S}\left[\hat{\Lambda}_{S} \otimes \hat{\rho}_{E}^{\text {pred }} \hat{\Gamma}_{j}\left(t_{p}\right)\right]}
$$

where the backward-evolved measurement device operator $\hat{\Gamma}_{j}(t)$ is given by (28), $\hat{\Lambda}_{S}$ is the sum of $\hat{\Lambda}_{i, S}$ and we have set the proportionality constant between the environmental preparation device operator and $\hat{\rho}_{E}^{\text {pred }}$ to unity because this is the a priori probability that the environment is prepared in this state. For our case, where the environment is not measured, the measurement device operator $\hat{\Gamma}_{j}$ will be proportional to $\hat{\Gamma}_{j, S} \otimes \hat{1}_{E}$. As $\hat{\Gamma}_{j}(t)$ evolves backwards in time towards $t_{p}$, it changes from being factorizable at $t_{m}$ to become in general more entangled.

We seek to express $P^{\Lambda \Gamma}(i \mid j)$ in terms of a reduced retrodictive density operator so that it has a form similar to (30), just as $P^{\Lambda \Gamma}(j \mid i)$ in (35) is similar to (27). The simplest way to do this is first to define a reduced backward evolved measurement device operator and then to normalize this so that the trace is unity. From (38) we see that we need a reduced backward-evolved measurement device operator in the form

$$
\hat{\Gamma}_{j, S}(t) \propto \operatorname{Tr}_{E}\left[\hat{\rho}_{E}^{\text {pred }} \hat{U}^{\dagger}\left(t_{m}, t\right) \hat{\Gamma}_{j, S} \otimes \hat{1}_{E} \hat{U}\left(t_{m}, t\right)\right] .
$$

We can now define a reduced retrodictive density operator for the system $S$ at the time $t$ as

$$
\hat{\rho}_{j, S}^{\mathrm{retr}}(t)=\frac{\hat{\Gamma}_{j, S}(t)}{\operatorname{Tr}_{S}\left[\hat{\Gamma}_{j, S}(t)\right]}
$$

Then by substituting (40) and (39) into (38) we can write the retrodictive probability in terms of operators acting on the state space of $S$ :

$$
P^{\Lambda \Gamma}(i \mid j)=\frac{\operatorname{Tr}_{S}\left[\hat{\Lambda}_{i, S} \hat{\rho}_{j, S}^{\mathrm{retr}}\left(t_{p}\right)\right]}{\operatorname{Tr}_{S}\left[\hat{\Lambda}_{S} \hat{\rho}_{j, S}^{\mathrm{retr}}\left(t_{p}\right)\right]} .
$$

Thus, if we know the reduced retrodictive density operator and the operation of the preparation device, we can calculate the retrodictive preparation probabilities by a formula of the same form (30) as for a closed system. For this, we wish to find an appropriate master equation governing the evolution backwards in time. We should note that the reduced predictive and retrodictive density operators in (34) and (40) are not simply related to each other and thus the master equation for the reduced retrodictive density operator is not immediately derivable from (37), for example simply by reversing the sign of the time. This, of course, is not due to some intrinsic time asymmetry in quantum mechanics; it arises from the asymmetry in the environmental boundary conditions. For both the predictive and retrodictive cases we are assuming that the environment is prepared in some particular state but not measured. To obtain symmetry we would have to assume in the retrodictive case that the environment is measured to be in some state and that we have no information about its preparation.

The most straightforward way of finding a retrodictive master equation for $\hat{\rho}_{j, S}^{\mathrm{retr}}(t)$ is to find an equation for the backwards evolution of $\hat{\Gamma}_{j, S}(t)$ and then use (40) to obtain the master equation. To do this, we use (26), the group property of the time-shift operator

$$
\hat{U}\left(t_{m}, t_{p}\right)=\hat{U}\left(t_{m}, t\right) \hat{U}\left(t, t_{p}\right),
$$

where $t$ is a time between $t_{m}$ and $t_{p}$, and the cyclic property of the trace to rewrite the numerator of the right side of (33) as

$$
\begin{array}{r}
\operatorname{Tr}_{E S}\left[\hat{U}\left(t, t_{p}\right) \hat{\rho}_{E}^{\text {pred }} \otimes \hat{\rho}_{i, S}^{\text {pred }} \hat{U}^{\dagger}\left(t, t_{p}\right) \hat{U}^{\dagger}\left(t_{m}, t\right) \hat{\Gamma}_{j, S}\right. \\
\left.\otimes \hat{1}_{E} \hat{U}\left(t_{m}, t\right)\right] .
\end{array}
$$

We leave the denominator of the right side of (33) as it is. Clearly $P^{\Lambda \Gamma}(j \mid i)$ does not vary as we change $t$ between $t_{p}$ and $t_{m}$ and so the derivative of the numerator (43) with respect to $t$ must vanish. Using the weak-coupling 
approximation (36) and (39) we can write (43) as proportional to

$$
\operatorname{Tr}_{S}\left[\hat{\rho}_{i, S}^{\mathrm{pred}}(t) \hat{\Gamma}_{j, S}(t)\right] .
$$

The vanishing of the derivative of this with respect to $t$ yields

$$
\operatorname{Tr}_{S}\left[\hat{\rho}_{i, S}^{\mathrm{pred}}(t) \dot{\hat{\Gamma}}_{j, S}(t)\right]=-\operatorname{Tr}_{S}\left[\dot{\hat{\rho}}_{i, S}^{\mathrm{pred}}(t) \hat{\Gamma}_{j, S}(t)\right] .
$$

Substituting (37) into (45) gives, after application of the cyclic property of the trace,

$$
\begin{aligned}
\operatorname{Tr}_{S}\left[\hat{\rho}_{i, S}^{\mathrm{pred}}(t) \dot{\hat{\Gamma}}_{j, S}(t)\right]= & \operatorname{Tr}_{S}\left(\hat { \rho } _ { i , S } ^ { \mathrm { pred } } ( t ) \left\{-i \hbar^{-1}\left[\hat{H}_{S}, \hat{\Gamma}_{j, S}(t)\right]\right.\right. \\
& -\sum_{q}\left[2 \hat{A}_{q}^{\dagger} \hat{\Gamma}_{j, S}(t) \hat{A}_{q}\right. \\
& -\hat{\Gamma}_{j, S}(t) A_{q}^{\dagger} \hat{A}_{q} \\
& \left.\left.\left.-\hat{A}_{q}^{\dagger} \hat{A}_{q} \hat{\Gamma}_{j, S}(t)\right]\right\}\right) .
\end{aligned}
$$

This is true for all $\hat{\rho}_{i, S}^{\text {pred }}(t)$, so the evolution equation for the reduced measurement device operator is

$$
\begin{aligned}
\dot{\hat{\Gamma}}_{j, S}(t)= & -i \hbar^{-1}\left[\hat{H}_{S}, \hat{\Gamma}_{j, S}(t)\right] \\
& -\sum_{q}\left[2 \hat{A}_{q}^{\dagger} \hat{\Gamma}_{j, S}(t) \hat{A}_{q}\right. \\
& \left.-\hat{\Gamma}_{j, S}(t) A_{q}^{\dagger} \hat{A}_{q}-\hat{A}_{q}^{\dagger} \hat{A}_{q} \hat{\Gamma}_{j, S}(t)\right] .
\end{aligned}
$$

We can then find the retrodictive master equation for $\hat{\rho}_{i S S}^{\text {retr }}(t)$ by substituting (47) into the time derivative of (40). This gives

$$
\begin{aligned}
\dot{\hat{\rho}}_{j, S}^{\text {retr }}(t)= & -i \hbar^{-1}\left[\hat{H}_{S}, \hat{\rho}_{j, S}^{\mathrm{retr}}(t)\right] \\
& -\sum_{q}\left[2 \hat{A}_{q}^{\dagger} \hat{\rho}_{j, S}^{\mathrm{retr}}(t) \hat{A}_{q}-\hat{\rho}_{j, S}^{\mathrm{retr}}(t) A_{q}^{\dagger} \hat{A}_{q}\right. \\
& \left.-\hat{A}_{q}^{\dagger} \hat{A}_{q} \hat{\rho}_{j, S}^{\mathrm{retr}}(t)\right] \\
& -2 \hat{\rho}_{j, S}^{\mathrm{retr}}(t) \operatorname{Tr}_{S}\left\{\hat{\rho}_{j, S}^{\mathrm{retr}}(t) \sum_{q}\left[\hat{A}_{q}^{\dagger}, \hat{A}_{q}\right]\right\}
\end{aligned}
$$

as the master equation for $\hat{\rho}_{j, S}^{\text {retr }}(t)$.

It is not difficult to see that (48) conserves the trace of $\hat{\rho}_{j, S}^{\text {retr }}(t)$, but (47) does not preserve the trace of $\hat{\Gamma}_{j, S}(t)$. The price of this preservation is that while (47) is a linear differential equation, the master equation (48) is more complicated. While (48) can be solved directly for some simple cases, the solution is not always obvious. In general, however, (48) is reducible to a linear equation by substituting a variable-trace operator

$$
\hat{B}(t)=\hat{\rho}^{\mathrm{retr}}(t) \exp \left(2 \int_{t_{m}}^{t} \operatorname{Tr}\left\{\hat{\rho}^{\mathrm{retr}}\left(t^{\prime}\right) \sum_{q}\left[\hat{A}_{q}^{\dagger}, \hat{A}_{q}\right]\right\} d t^{\prime}\right),
$$

which gives a linear equation for $\hat{B}(t)$. Then $\hat{\rho}^{\text {retr }}(t)$ can be recovered from the solution as $\hat{B}(t) / \operatorname{Tr} \hat{B}(t)$. The linear equation obtained, however, is the same as (47) so
$\hat{B}(t)$ is just proportional to $\hat{\Gamma}_{j, S}(t)$. It is thus more convenient in general to calculate retrodictive probabilities by solving (47) for $\hat{\Gamma}_{j, S}(t)$ and then using

$$
P^{\Lambda \Gamma}(i \mid j)=\frac{\operatorname{Tr}_{S}\left[\hat{\Lambda}_{i, S} \hat{\Gamma}_{j, S}\left(t_{p}\right)\right]}{\operatorname{Tr}_{S}\left[\hat{\Lambda}_{S} \hat{\Gamma}_{j, S}\left(t_{p}\right)\right]}
$$

which is derivable from (39), (38) and (28), instead of solving (48) for $\hat{\rho}_{j, S}^{\text {retr }}(t)$ and using (41).

To give a physical interpretation of $\hat{\Gamma}_{j, S}(t)$, we first show that this is a non-negative operator for all $t$ between $t_{p}$ and $t_{m}$ as follows. Consider an operator $\hat{\sigma}(t)$, acting on the state space of $S$, defined to obey the predictive master equation (37) for times $t$ between (and including) $T$ and $t_{m}$ where $T$ is some time such that $t_{p} \leq T \leq t_{m}$. Let $\hat{\sigma}(T)$ $=|u\rangle\langle u|$ where $|u\rangle$ is some arbitrary pure state of $S$. Then $\hat{\sigma}(t)$ will have the form of a predictive density operator and so $\hat{\sigma}\left(t_{m}\right)$ will be non-negative definite. From (39), $\hat{\Gamma}_{j, S}\left(t_{m}\right)$ is just $\hat{\Gamma}_{j, S}$, which is also non-negative definite. Thus

$$
\operatorname{Tr}_{S}\left[\hat{\sigma}\left(t_{m}\right) \hat{\Gamma}_{j, S}\left(t_{m}\right)\right] \geq 0 .
$$

It is not difficult to show directly from (37) with $\hat{\sigma}(t)$ in place of $\hat{\rho}_{i, S}^{\text {pred }}(t)$ and from (47) that the time derivative of $\operatorname{Tr}_{S}\left[\hat{\sigma}(t) \hat{\Gamma}_{j, S}(t)\right]$ is zero for $t$ between $T$ and $t_{m}$. Consequently

$$
\operatorname{Tr}_{S}\left[\hat{\sigma}(T) \hat{\Gamma}_{j, S}(T)\right] \geq 0 .
$$

Writing the left side of (52) as $\left\langle u\left|\hat{\Gamma}_{j, S}(T)\right| u\right\rangle$ shows that $\hat{\Gamma}_{j, S}(T)$ is non-negative definite for all times $T$ between $t_{p}$ and $t_{m}$.

The non-negativity of $\hat{\Gamma}_{i, S}(t)$ has two consequences. Firstly it follows from (40) that $\hat{\rho}_{j, S}^{\text {retr }}(t)$ is also nonnegative. This, combined with the trace of $\hat{\rho}_{j, S}^{\text {retr }}(t)$ being conserved as unity, means that (48) is a legitimate master equation. The second consequence is that $\hat{\Gamma}_{j, S}(t)$ can be considered to be measurement device operators associated with measurement events $j$. Furthermore, if $\hat{\Gamma}_{j, S}(t)$ sum to be proportional to the unit operator on the state space of $S$ it follows from (47) that this sum is also conserved. Thus if the set of operators $\hat{\Gamma}_{j, S}\left(t_{m}\right)$, which are proportional to $\hat{\Gamma}_{j, S} \otimes \hat{1}_{E}$, describes the operation of an unbiased measuring device, so too will the set of operators $\hat{\Gamma}_{j, S}(t)$ and thus also $\hat{\Gamma}_{j, S}\left(t_{m}\right)$. Consequently the open system considered here allows a similar interpretation as does the closed system equation (29) with the measurement, or collapse of the state, taking place immediately after the preparation time $t_{p}$. It should be noted that in the common interpretation, in which the operation of the measurement device is described by $\hat{\Gamma}_{j, S}\left(t_{m}\right)$, the initial environment state is considered to be part of the description of the operation of the preparation device. For the new interpretation, with the measurement taking place at $t_{p}$ in accord with (50), the initial environment state is considered to be part of the description of the 
operation of the measurement device. This is analogous to a homodyne detection system in which the initial state of the local oscillator is considered to be part of the operation of the measurement device rather than part of the operation of the preparation device along with the signal field state. The conservation of non-negativity allows us, in fact, to interpret (27) and (29) in terms of the measurement or collapse taking place at any time $t$ between $t_{p}$ and $t_{m}$ with non-negative preparation and measurement device operators defined appropriately. The physical interpretation of the invariance of $P^{\Lambda \Gamma}(j \mid i)$ under changes of $t$ is that measurable probabilities are independent of when we choose the collapse time. This underlines the somewhat arbitrary nature of this concept.

By comparing the predictive and retrodictive master equations (37) and (48), we see that when the interaction with the environment can be ignored, for example for short enough time intervals, the evolution is given by just the first term in each. The two equations for this unitary evolution are then the same. It should be remembered, however, that we have defined the time $t$ so that it increases in both cases. For retrodiction, it is more natural to define a premeasurement time as

$$
\tau=t_{m}-t
$$

so that

$$
d \hat{\rho}_{j, S}^{\mathrm{retr}}(\tau) / d \tau=-\dot{\hat{\rho}}_{j, S}^{\mathrm{retr}}(t)
$$

The unitary part of the retrodictive master equation in terms of $\tau$ is then seen explicitly to be the time inverse of the corresponding part of the predictive master equation. That the complete retrodictive master equation is not simply the time inverse of the predictive equation is illustrated by the following. If there is an extremely long time between preparation and measurement, both $\hat{\rho}_{j, S}^{\text {pred }}(t)$ and $\hat{\rho}_{j, S}^{\text {retr }}(\tau)$ can reach their steady-state values for which $\dot{\hat{\rho}}_{j, S}^{\text {pred }}(t)$ and $d \hat{\rho}_{j, S}^{\text {retr }}(\tau) / d \tau$ are zero. A solution of the resulting steady-state retrodictive equation is clearly $\hat{\rho}_{j, S}^{\text {retr }}(\infty)=\hat{1}_{S} / D$ where $\hat{1}_{S}$ is the unit operator acting on the state space of $S$ and $D$ is the dimension of that space. This represents the no-information state. Essentially, at the measurement time the retrodictive state is the product of the measured state and the no-information environment state. As we go backwards in time from the measurement, the retrodictive system state becomes more entangled with the environment and we lose information about the system state. In the limit of very long times in the past, the system state becomes completely unretrodictable. There is no similar simple general solution of the predictive master equation, however, which would imply unpredictability for long times. Indeed for the case of an excited two-level atom undergoing spontaneous emission into the environmental vacuum field, for example, it becomes very likely that the atom will be found in its ground state in the long term future, an outcome which is very predictable.

\section{COHERENTLY DRIVEN ATOM}

\section{A. Retrodictive density operator}

As an example of a retrodictive master equation and its solution, we examine the case of a two-level atom driven on resonance by a strong laser field. The solution of the predictive master equation for this system is well known [13], with the atom exhibiting damped Rabi oscillations at a frequency $\Omega=\left(V^{2}-\gamma^{2} / 4\right)^{1 / 2}$ where $V$ is proportional to the strength of the laser field and $\gamma$ is the spontaneous decay rate. We can write the Hamiltonian describing the interaction between the atom and the laser field in the interaction picture in the semiclassical form

$$
\hat{H}=\frac{\hbar}{2} V\left(\hat{\sigma}_{+}+\hat{\sigma}_{-}\right)
$$

where $\sigma_{+}=|e\rangle\langle g|$ and $\sigma_{-}=|g\rangle\langle e|$. A comparison of (37) with the predictive master equation for this system [13], that is,

$$
\begin{aligned}
\dot{\hat{\rho}}_{i, S}^{\text {pred }}(t)= & -\frac{i V}{2}\left[\left(\hat{\sigma}_{+}+\hat{\sigma}_{-}\right), \hat{\rho}_{i, S}^{\text {pred }}(t)\right] \\
& +\gamma\left[2 \hat{\sigma}_{-} \hat{\rho}_{i, S}^{\text {pred }}(t) \hat{\sigma}_{+}-\hat{\sigma}_{+} \hat{\sigma}_{-} \hat{\rho}_{i, S}^{\text {pred }}(t)\right. \\
& \left.-\hat{\rho}_{i, S}^{\text {pred }}(t) \hat{\sigma}_{+} \hat{\sigma}_{-}\right],
\end{aligned}
$$

shows that $q$ in (37) has only one value, with $A_{q}=$ $\gamma^{1 / 2} \sigma_{-}$. Thus, using $\hat{B}(\tau)$ in place of $\hat{\Gamma}_{j, S}(\tau)$, we can write the linear form (47) of the retrodictive master equation in terms of the premeasurement time $\tau$ as

$$
\begin{aligned}
d \hat{B}(\tau) / d \tau= & \frac{i V}{2}\left[\left(\hat{\sigma}_{+}+\hat{\sigma}_{-}\right), \hat{B}(\tau)\right] \\
& +\gamma\left[2 \hat{\sigma}_{+} \hat{B}(\tau) \hat{\sigma}_{-}-\hat{B}(\tau) \hat{\sigma}_{+} \hat{\sigma}_{-}\right. \\
& \left.-\hat{\sigma}_{+} \hat{\sigma}_{-} \hat{B}(\tau)\right] .
\end{aligned}
$$

We can solve (57) by converting it to a set of c-number equations. We write

$$
\hat{B}(\tau)=u(\tau) \hat{\sigma}_{1}+v(\tau) \hat{\sigma}_{2}+w(\tau) \hat{\sigma}_{3}+x(\tau) \hat{1}_{S}
$$

where $\hat{1}_{S}$ is the unit operator acting on the space of the atom and

$$
\begin{gathered}
\hat{\sigma}_{1}=\hat{\sigma}_{+}+\hat{\sigma}_{-} \\
\hat{\sigma}_{2}=-i\left(\hat{\sigma}_{+}-\hat{\sigma}_{-}\right) \\
\hat{\sigma}_{3}=2 \hat{\sigma}_{+} \hat{\sigma}_{-}-\hat{1}_{S} .
\end{gathered}
$$

Then substitution of (58) into (57) yields

$$
\begin{aligned}
& \frac{d u}{d \tau} \hat{\sigma}_{1}+\frac{d v}{d \tau} \hat{\sigma}_{2}+\frac{d w}{d \tau} \hat{\sigma}_{3}+\frac{d x}{d \tau} \hat{1}_{S}= \\
& \quad-\gamma u \hat{\sigma}_{1}-(\gamma v-V w) \hat{\sigma}_{2}-(V v+2 \gamma w) \hat{\sigma}_{3}-2 \gamma w \hat{1}(\$ 62)
\end{aligned}
$$


Multiplying (62) by $\hat{\sigma}_{1}, \hat{\sigma}_{2}$ and $\hat{\sigma}_{3}$ respectively and taking the trace of both sides gives

$$
\begin{gathered}
\frac{d u}{d \tau}=-\gamma u \\
\frac{d v}{d \tau}=-\gamma v+V w \\
\frac{d w}{d \tau}=-V v-2 \gamma w
\end{gathered}
$$

and taking the trace of both sides of (62) gives

$$
\frac{d x}{d \tau}=-2 \gamma w
$$

The solution of (63) is simply

$$
u(\tau)=u(0) \exp (-\gamma \tau) .
$$

The simultaneous equations (64) and (65) are straightforwardly solved by standard means 15 to yield

$$
\begin{aligned}
v(\tau)= & \exp (-3 \gamma \tau / 2)\{v(0)[\cos (\Omega \tau) \\
& \left.+\gamma(2 \Omega)^{-1} \sin (\Omega \tau)\right] \\
& \left.+w(0) V \Omega^{-1} \sin (\Omega \tau)\right\}
\end{aligned}
$$

and

$$
\begin{aligned}
w(\tau)= & \exp (-3 \gamma \tau / 2)\left\{-v(0) V \Omega^{-1} \sin (\Omega \tau)\right. \\
& \left.+w(0)\left[\cos (\Omega \tau)-\gamma(2 \Omega)^{-1} \sin (\Omega \tau)\right]\right\}
\end{aligned}
$$

which allows us to find $x$ from 66 as

$$
\begin{aligned}
x(\tau)= & x(0)+\frac{2 \gamma[V v(0)-\gamma w(0)]}{2 \gamma^{2}+V^{2}} \\
& +\exp (-3 \gamma \tau / 2) \frac{-2 \gamma}{2 \gamma^{2}+V^{2}} \\
& \times\left\{\frac{\left(\gamma^{2}+2 V^{2}\right) w(0)+3 \gamma V v(0)}{2 \Omega} \sin (\Omega \tau)\right. \\
& +[V v(0)-\gamma w(0)] \cos (\Omega \tau)\} .
\end{aligned}
$$

Substituting (67), 68), 69) and (70) into (58) gives $\hat{B}(\tau)$. The retrodictive density operator is then just

$$
\hat{\rho}_{j, S}^{\mathrm{retr}}(\tau)=\frac{\hat{B}(\tau)}{2 x(\tau)},
$$

which has unit trace as required.

\section{B. Some detection events}

As a check of the retrodictive density operator (71) obtained from our general retrodictive master equation, we now calculate its matrix elements for some specific detection events. These can be compared with corresponding matrix elements in [16] calculated by a quite different method involving solutions of the predictive master equation and specific measurement POM elements.

As our first example, suppose the atom is detected at time $\tau=0$ in the excited state $|e\rangle\langle e|$. The measurement device operator corresponding to this detection event is proportional to $|e\rangle\langle e|$, which is also the retrodictive density operator at this time. Thus $\hat{B}(0) \propto|e\rangle\langle e|$. We note that it is not necessary to say whether or not the measurement device operator is an element of a POM. By writing $|e\rangle\langle e|$ as $\left(1+\hat{\sigma}_{3}\right) / 2$ we see that $u(0)$ and $v(0)$ are both zero and $w(0)=x(0)$. With these values we find that

$$
u(\tau)=0
$$

$$
v(\tau)=w(0) \exp (-3 \gamma \tau / 2) V \Omega^{-1} \sin (\Omega \tau)
$$

$$
w(\tau)=w(0) \exp (-3 \gamma \tau / 2)\left[\cos (\Omega \tau)-\gamma(2 \Omega)^{-1} \sin (\Omega \tau)\right]
$$

and

$$
\begin{aligned}
x(\tau)= & \frac{w(0)}{2 \gamma^{2}+V^{2}}\left\{V^{2}-2 \gamma \exp (-3 \gamma \tau / 2)\right. \\
& \left.\times\left[\frac{\left(\gamma^{2}+2 V^{2}\right)}{2 \Omega} \sin (\Omega \tau)-\gamma \cos (\Omega \tau)\right]\right\} .
\end{aligned}
$$

The matrix elements of $\hat{\rho}_{j, S}^{\text {retr }}(\tau)$ are easily found from (71) and (58) to be

$$
\begin{aligned}
\left\langle e\left|\hat{\rho}_{j, S}^{\mathrm{retr}}(\tau)\right| g\right\rangle & =\frac{u(\tau)-i v(\tau)}{2 x(\tau)} \\
\left\langle e\left|\hat{\rho}_{j, S}^{\mathrm{retr}}(\tau)\right| e\right\rangle & =\frac{w(\tau)+x(\tau)}{2 x(\tau)}
\end{aligned}
$$

with $\left\langle g\left|\hat{\rho}_{j, S}^{\text {retr }}(\tau)\right| e\right\rangle=\left\langle e\left|\hat{\rho}_{j, S}^{\text {retr }}(\tau)\right| g\right\rangle^{*}$ and $\left\langle g\left|\hat{\rho}_{j, S}^{\text {retr }}(\tau)\right| g\right\rangle=$ $1-\left\langle e\left|\hat{\rho}_{j, S}^{\text {retr }}(\tau)\right| e\right\rangle$. From (72), (73) and (74) we obtain

$$
\left\langle e\left|\hat{\rho}_{j, S}^{\mathrm{retr}}(\tau)\right| g\right\rangle=\frac{-i V w(0)}{2 x(\tau) \Omega} \exp (-3 \gamma \tau / 2) \sin (\Omega \tau)
$$

$$
\begin{aligned}
\left\langle e\left|\hat{\rho}_{j, S}^{\mathrm{retr}}(\tau)\right| e\right\rangle= & \frac{w(0)}{2 x(\tau)\left(V^{2}+2 \gamma^{2}\right)}\left\{V^{2}\right. \\
& +\exp (-3 \gamma \tau / 2)\left[\left(V^{2}+4 \gamma^{2}\right) \cos (\Omega \tau)\right. \\
& \left.\left.-\frac{\gamma}{2 \Omega}\left(5 V^{2}+4 \gamma^{2}\right) \sin (\Omega \tau)\right]\right\} .
\end{aligned}
$$

These are identical to the corresponding expressions derived in $[16$ except that here we have $x(\tau) / w(0)$ in place of the normalization factor $N$. As we already have an expression for this in (75) there is no need to calculate it separately as we had to in [16]. These results therefore not only confirm the validity of our new method but also display its advantages.

The Rabi oscillations are clearly evident in both the diagonal and off-diagonal elements of the density matrix. 
In the limit of long $\tau$, that is, in the distant past, the off-diagonal elements vanish and both diagonal elements tend to $1 / 2$. This is the density matrix describing the noinformation state discussed earlier. The actual state the atom was prepared in is thus essentially unretrodictable for these long times. This contrasts with the predictive density matrix, which also exhibits Rabi oscillations for short times but for long times in the future tends to an equilibrium state which is not the no-information state, instead it is determined by the relative value of $V$ and $\gamma$ [13.

If the atom is detected in its ground state, we simply take $u(0)$ and $v(0)$ as zero and $w(0)=-x(0)$ and obtain a slight variation on the above elements of the retrodictive density matrix.

If the atom is detected in the superposition state $2^{-1 / 2}(|e\rangle+|g\rangle)$, the measurement device operator is proportional to $\left(1+\hat{\sigma}_{1}\right)$, so $v(0)$ and $w(0)$ are zero and $u(0)$ $=x(0)$. Then we obtain simply

$$
\begin{aligned}
u(\tau) & =u(0) \exp (-\gamma \tau) \\
v(\tau) & =0 \\
w(\tau) & =0 \\
x(\tau) & =u(0)
\end{aligned}
$$

The retrodictive density operator is then easily found from (71) and (58) to be

$$
\hat{\rho}_{j, S}^{\text {retr }}(\tau)=\left[1+\hat{\sigma}_{1} \exp (-\gamma \tau)\right] / 2 .
$$

This has matrix elements

$$
\begin{aligned}
\left\langle e\left|\hat{\rho}_{j, S}^{\text {retr }}(\tau)\right| g\right\rangle & =[\exp (-\gamma \tau)] / 2 \\
\left\langle e\left|\hat{\rho}_{j, S}^{\text {retr }}(\tau)\right| e\right\rangle & =1 / 2
\end{aligned}
$$

which are precisely those calculated in $[16$. Because of the phase of the detected state there are no Rabi oscillations, the density operator (84) simply decays to the no-information state in the infinite past. To obtain Rabi oscillations in retrodiction from a detected state that is an equal superposition of $|e\rangle$ and $|g\rangle$, we could instead use a measuring device that detects the state $2^{-1 / 2}(|e\rangle+i|g\rangle)$, for which the measurement device operator is $\left(1+\hat{\sigma}_{2}\right) / 2$ so $v(0)$ is not zero. Some of the oscillatory terms in 68, (69) and (70) are then retained.

\section{Preparation probabilities}

The elements of the retrodictive density matrix, diagonal or off-diagonal, do not translate into preparation probabilities until we specify the operation of the preparation device, that is, the values of $\hat{\Lambda}_{i, S}$ for use with 41. The simplest calculations of preparation probabilities are those in the infinite- $\tau$ limit. As we have seen, in this limit the retrodictive density operator becomes proportional to the unit operator. Substituting this into (41) yields

$$
P^{\Lambda \Gamma}(i \mid j)=\frac{\operatorname{Tr}_{S} \hat{\Lambda}_{i, S}}{\operatorname{Tr}_{S} \hat{\Lambda}_{S}},
$$

which from (8) and (10) is seen to be just the a priori probability for the preparation event $i$. Thus no further information has been gained from knowing the measurement event.

We consider a preparation device which can prepare the two-level atom in a pure state $|i\rangle$ that is either $|e\rangle$, $|g\rangle$ or some superposition of the two. One might envisage a device that can apply pulses of resonant coherent radiation of various durations and phases to an atom in its ground state. The application of a $\pi$ pulse would prepare the atom its the excited state and a $\pi / 2$ pulse with appropriate phase would prepare the state $2^{-1 / 2}(|e\rangle+|g\rangle)$. A zero pulse would prepare the atom in the ground state. The operation of such a device is described by a set of preparation device operators, each of which represents a possible prepared state and incorporates the a priori probability for that state to be prepared. That is $\hat{\Lambda}_{i, S}$ is proportional to $P^{\Lambda}(i)|i\rangle\langle i|$. Clearly, even if the prepared states are restricted to $|e\rangle$ and $|g\rangle$ the preparation device operators will not be the elements of a POM unless these are produced with equal a priori probabilities. If the device can only prepare the states $2^{-1 / 2}(|e\rangle+|g\rangle)$ and $|g\rangle$, the preparation device operators are not proportional to the elements of a POM, whatever each a priori probability is. This is the situation for most preparation devices and can be contrasted with measurement devices, very many of whose operations can be described by a POM.

To be specific, let us assume that the preparation device is unbiased, preparing the atom in states $|e\rangle$ and $|g\rangle$ with equal probability. Then $\hat{\Lambda}_{e, S}=|e\rangle\langle e| / 2$ and $\hat{\Lambda}_{g, S}=$ $|g\rangle\langle g| / 2$ and the preparation probabilities for states $|e\rangle$ and $|g\rangle$ become, from (41), simply the diagonal elements $\left\langle e\left|\hat{\rho}_{j, S}^{\text {retr }}(\tau)\right| e\right\rangle$ and $\left\langle g\left|\hat{\rho}_{j, S}^{\text {retr }}(\tau)\right| g\right\rangle$. On the other hand, for a biased device that prepares the atom in states $|e\rangle$ and $|g\rangle$ with a priori probabilities $p$ and $1-p$ respectively, $\hat{\Lambda}_{e, S}$ and $\hat{\Lambda}_{g, S}$ are $p|e\rangle\langle e|$ and $(1-p)|g\rangle\langle g|$. Then the probability that the atom was prepared in the excited state if the measurement is $j$ is

$$
P^{\Lambda \Gamma}(e \mid j)=\frac{p\left\langle e\left|\hat{\rho}_{j, S}^{\mathrm{retr}}(\tau)\right| e\right\rangle}{p\left\langle e\left|\hat{\rho}_{j, S}^{\mathrm{retr}}(\tau)\right| e\right\rangle+(1-p)\left\langle g\left|\hat{\rho}_{j, S}^{\mathrm{retr}}(\tau)\right| g\right\rangle} .
$$

In the limit of long $\tau$, this expression becomes equal to $p$, the a priori probability that the atom was prepared in the excited state, as discussed above. For short times $\tau$, the dependence of the probability (88) on $\tau$ will exhibit an oscillatory behavior depending on the state detected.

A simple but interesting case is for an unbiased preparation device that prepares the states $|+\rangle=2^{-1 / 2}(|e\rangle+$ $|g\rangle)$ and $|-\rangle=2^{-1 / 2}(|e\rangle-|g\rangle)$ with equal probability. The preparation device operators are $|+\rangle\langle+| / 2$ and $|-\rangle\langle-| / 2$. The preparation probabilities for $|+\rangle$ and $|-\rangle$ if the measurement event is $j$ are, from (41), just $\left\langle+\left|\hat{\rho}_{j, S}^{\text {retr }}(\tau)\right|+\right\rangle$ and $\left\langle-\left|\hat{\rho}_{j, S}^{\text {retr }}(\tau)\right|-\right\rangle$ respectively. For the case discussed earlier in which the atom is detected in the superposition state $2^{-1 / 2}(|e\rangle+|g\rangle)$ and so the retrodictive density operator is given by (34), we find that these probabilities are just $[1+\exp (-\gamma \tau)] / 2$ and $[1-\exp (-\gamma \tau)] / 2$ respectively. The 
values of the probabilities change from unity and zero for small $\tau$ to both being one half for large $\tau$ and do not exhibit oscillations at any time. Thus the decay from the measured state to the no-information state is shown explicitly as the only change with time involved.

The above examples illustrate the procedure involved and the preparation probabilities for preparation devices with other operations are easily calculated from (41).

\section{CONCLUSION}

The emerging importance of quantum communication and quantum cryptography has made it worthwhile to re-investigate quantum retrodiction as a means of solving the basic quantum communication problem. This involves calculating the probabilities that particular states were prepared when various states are detected. Where original investigations of quantum retrodiction were in terms of closed systems, quantum communication is more likely to involve open systems because of interaction with the environment. For closed systems the evolution is unitary, so the backward-time evolution equation for the state from measurement to preparation is just the simple inverse of the forward-time equation. For open systems the situation is not so simple. When a system is weakly coupled to a large environment, the forward-time evolution is given by a master equation based on knowledge of the prepared state of the system and the initial state of the environment. In the retrodictive situation, the measured state of the system is known, but not the final state of the environment because this is not measured. Instead, the initial state of the environment is known. Thus the backward-time evolution equation will not be the simple inverse of the forward-time master equation. In this paper we have derived the general, that is Lindblad, form of the backward-time, or retrodictive, master equation. We also prove that the retrodictive density operator remains non-negative definite at all times between measurement and preparation. As well as confirming the legitimacy of the master equation as an equation for the a retrodictive density operator, the non-negativity also allows us to interpret the evolution plus the measurement in terms of the operation of another measurement device. This shows that we can consider the measurement, or collapse of the state, as taking place immediately after the preparation if we wish. Indeed it is possible to have a consistent interpretation of the preparation-measurement process with the collapse taking place at any time between preparation and measurement, showing the somewhat arbitrary nature of the concept. The vanishing of the time derivative of (44), or in general of (43), effectively incorporates the invariance of the physically measurable probability $P^{\Lambda \Gamma}(j \mid i)$ with choice of collapse time.

The retrodictive master equation is not in general linear, underlining the fact that it is not the simple inverse of the predictive master equation. We have shown, however, that it can always be linearized by means of a suitable substitution, allowing analytical solutions for particular systems. As an explicit example, we have considered retrodiction for an atom driven by a coherent optical field and have solved the master equation for the general retrodictive density operator at all times between measurement and preparation. This allows us to select any particular measurement event we wish and easily find the probabilities of various possible preparation events based on this knowledge. Where the results overlap with previous calculations based on an entirely different, and less direct, method [16] there is perfect agreement, which serves as confirmation of the validity of our new approach.

As we have discussed previously [4], preparation probabilities can always be found from predictive evolution plus inference by means of Bayes' theorem. Retrodictive quantum mechanics, however, offers a more direct and often simpler means of finding these probabilities as well as giving a different insight into the interpretation of the quantum state. In this paper we have extended the theory of quantum retrodiction for open systems by deriving of a general retrodictive master equation.

\section{Acknowledgments}

We thank the United Kingdom Engineering and Physical Sciences Research Council, the Australian Research Council, the Royal Society of Edinburgh and the Scottish Executive Education and Lifelong Learning Department for financial support. We also thank N. Lütkenhaus for useful discussions.
[1] J. von Neumann, Mathematical Foundations of Quantum Mechanics (Princeton University Press, Princeton, 1955).

[2] C. W. Helstrom, Quantum Detection and Estimation Theory (Academic Press, New York, 1976).

[3] Y. Aharonov, P. G. Bergman and J. L. Lebowitz, Phys. Rev. 134, B1410 (1964).

[4] S. M. Barnett, D. T. Pegg, and J. Jeffers, J. Mod. Opt. 47, 1779 (2000).

[5] R. H. Penfield, Am. J. Phys. 34, 422 (1966); Y. Aharonov and D. Z. Albert, Phys. Rev. D 29, 223 (1984); Y.
Aharonov and D. Z. Albert, Phys. Rev. D 29, 228 (1984); Y. Aharonov and L. Vaidman, J. Phys. A: Math. Gen. 24, 2315 (1991); D. T. Pegg and S. M. Barnett, Quantum and Semiclass. Opt. 1, 442 (1999); F. J. Belinfante, Measurements and Time Reversal in Objective Quantum Theory (Pergamon Press, Oxford,1975). S. M. Barnett, D. T. Pegg, J. Jeffers, O. Jedrkiewicz and R. Loudon Phys. Rev. A 62, 022313 (2000).

[6] Quantum Communication, Computing and Measurement, edited by O. Hirota, A. S. Holevo and C. M. Caves 
(Plenum, New York, 1997)

[7] S. J. D. Phoenix and P. D. Townsend, Contemp. Phys. 36, 165 (1995) and references therein; D. Bouwmeester, A. Ekert and A. Zielinger (eds.), The Physics of Quantum Information (Springer, Berlin, 2000) and references therein.

[8] S. M. Barnett, D. T. Pegg, J. Jeffers, and O. Jedrkiewicz, Phys. Rev. Lett. 86, 2455 (2001).

[9] D. T. Pegg, S. M. Barnett, and J. Jeffers, J. Mod. Opt. (in press).

[10] M. L. Boas, Mathematical Methods in the Physical Sciences (Academic Press, New York, 1983), p. 695.

[11] G. E. P. Box and G. C. Tiao, Bayesian Inference in Statistical Analysis (Addison-Wesley, Sydney, 1973), p. 10.

[12] C. H. Bennett and G. Brassard, Proceedings of the IEEE
International Conference on Computers, Systems and Signal Processing (Bangalore, 1984), p. 175.

[13] See, for example, S. M. Barnett and P. M. Radmore Methods in Theoretical Quantum Optics (Oxford University Press, Oxford, 1997).

[14] G. Lindblad, Commun. Math. Phys. 48, 119 (1976); S. Stenholm, in Quantum Dynamics of Simple Systems, edited by G.-L. Oppo, S. M. Barnett, E. Riis, and M. Wilkinson (SUSSP Publications, Edinburgh, 1996) p. 299.

[15] See, for example, D. H. Menzel, Fundamental Formulas of Physics (Dover Publications, New York, 1960) p. 36.

[16] S. M. Barnett, D. T. Pegg, J. Jeffers, and O. Jedrkiewicz, J. Phys. B, At. Mol. Opt. Phys. 33, 3047 (2000). 